\title{
Achieving thermally conductive low loss PVDF-based dielectric composites via surface functionalization and orientation of $\mathrm{SiC}$ nanowires
}

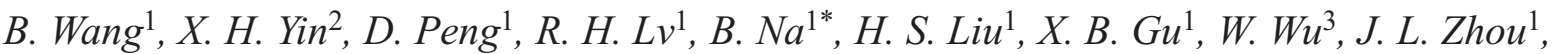 \\ Y. Zhang $^{1}$ \\ ${ }^{1}$ Jiangxi Province Key Laboratory of Polymer Micro/Nano Manufacturing and Devices, School of Chemistry, Biology and \\ Materials Science, East China University of Technology, 330013 Nanchang, P. R. China \\ ${ }^{2}$ College of Urban Transportation and Logistics, Shenzhen Technology University, 518118 Shenzhen, P. R. China \\ ${ }^{3}$ Key Laboratory of Polymer Processing Engineering of Ministry of Education, South China University of Technology, \\ 510640 Guangzhou, P. R. China
}

Received 4 May 2019; accepted in revised form 17 July 2019

\begin{abstract}
Semi-conductive silicon carbide $(\mathrm{SiC})$ nanowires were amino-functionalized to achieve better dispersion in poly(vinylidene fluoride) (PVDF) matrix. It was found that PVDF based composites with amino-functionalized $\mathrm{SiC}$ (f-SiC) nanowires exhibited lower loss tangent than their counterparts with bare SiC nanowires, especially at a filler loading of $13.8 \mathrm{vol} \%$. The loss tangent at $1 \mathrm{kHz}$ of PVDF/f-SiC nanowires $(86.2 / 13.8, \mathrm{v} / \mathrm{v})$ composite is only 0.048 , which is nearly one quarter of that of its counterpart with bare SiC nanowires. The nearly one order of magnitude lower AC conductivity at $1 \mathrm{kHz}$ is responsible for the remarkable decrease of the loss tangent, since the interlacing of $\mathrm{f}-\mathrm{SiC}$ nanowires was avoided via their parallel orientation, facilitated by the enhanced interfacial interaction. In addition to the low loss, the PVDF/f-SiC nanowires $(86.2 / 13.8, \mathrm{v} / \mathrm{v})$ composite exhibited about twofold increase of the dielectric permittivity at $1 \mathrm{kHz}$, compared to neat PVDF. Moreover, the thermal conductivity of PVDF/f-SiC nanowires (86.2/13.8, v/v) composite was increased to twice that of neat PVDF. The thermally conductive, high dielectric permittivity, and low loss PVDF/f-SiC nanowires composites may find potential applications in capacitors for microelectronics.
\end{abstract}

Keywords: polymer composites, interfacial interaction, dielectric property, thermal conductivity

\section{Introduction}

Poly(vinylidene fluoride) (PVDF) exhibits relatively high dielectric permittivity and breakdown strength among polymer materials [1,2]. Moreover, as a polymer material, PVDF shows the merits of good flexibility, good processability, and relatively low cost. As a result, PVDF-based dielectric nanocomposites have drawn much attention in both academia and industry on pursuing high dielectric permittivity and low loss properties for the potential applications in flexible energy storage and electronic devices [3-6].
To enhance the dielectric permittivity of PVDF, a common way is to homogeneously disperse nonconductive fillers (such as barium titanate [7-9], titanium dioxide $[10,11]$, and metal chloride $[12,13]$, etc.) into the PVDF matrix. The loss tangent can be usually maintained at a low value approximating that of neat PVDF due to the nonconductive nature of ceramic nanofillers. However, a large amount of nonconductive fillers (e.g. higher than $30 \mathrm{vol} \%$ ) are needed to achieve an obvious increase of the dielectric permittivity $[14,15]$. High filler loadings will inevitably 
deteriorate the processability, flexibility, and breakdown strength of the PVDF-based nanocomposites. Moreover, it is still a challenge to homogeneously disperse the nonconductive fillers at high loadings.

Alternatively, the dielectric permittivity can be largely improved via adding conductive nanofillers (such as metal nanoparticles [16, 17], carbon nanotubes (CNTs) [18, 19], and graphene [20,21], etc.) into the PVDF matrix. The dielectric permittivity can be several orders higher than that of neat PVDF as the loading of conductive nanofillers is approaching (but still lower than) the percolation threshold. Thus, the needed loading of conductive nanofillers to achieve high dielectric permittivity is usually far lower than that of nonconductive fillers. These PVDF-based nanocomposites, however, usually exhibit high dielectric loss, since the conductive nanofillers will inevitably cause large amounts of free charges. Moreover, the local conductive path may form due to the tunneling effect between adjacent conductive nanofillers [22]. One of the effective ways to solve this issue is to decorate a layer of insulating shell onto the surface of conductive nanofillers [23, 24]. Another effective way is to align the conductive nanofillers (such as CNTs) by anchoring them on a template [25]. Despite effectiveness in lowering the dielectric loss, these strategies are usually achieved via complicated chemical synthesis, and thus the productivity is relatively low.

Recently, the semi-conductive silicon carbide ( $\mathrm{SiC})$ nanowires attracted the attention as high dielectric permittivity fillers, due to their high density of stacking faults [26]. It is reported that the dielectric permittivity of PVDF is remarkably increased via the addition of $\mathrm{SiC}$ nanowires [27, 28]. However, the $\mathrm{SiC}$ nanowires tend to interlace with each other at a slightly higher loading, due to their high aspect ratios. This will lead to the significant increase of leakage current, and thus the increase of dielectric loss. From the view of polymer processing, the interlacing of $\mathrm{SiC}$ nanowires might be avoided by orienting them parallelly in the polymer matrix. Thus, the authors are inspired to prepare $\mathrm{PVDF} / \mathrm{SiC}$ nanowires composites with oriented structures, and to facilitate the orientation of $\mathrm{SiC}$ nanowires via surface functionalization.

In this work, the $\mathrm{SiC}$ nanowires were amino-functionalized by a simple ball milling strategy. The aminofunctionalized $\mathrm{SiC}$ (f-SiC) nanowires were observed to be more compatible with the PVDF matrix, and more prone to orient under the compression flow during the hot pressing. Thus, the interlacing of SiC nanowires was avoided, and a low dielectric loss $(0.048)$ at $1 \mathrm{kHz}$ was achieved even at a relatively high loading (13.8 vol\%). Moreover, the thermal conductivity was simultaneously improved due to the presence of $\mathrm{SiC}$ nanowires.

\section{Experimental}

\subsection{Materials}

SiC nanowires (XF J54) were purchased from Xianfeng Nano Technology Co., Ltd, Nanjing, China. The PVDF resin (solef 6010) with a melt flow index of $6.0 \mathrm{~g} / 10 \mathrm{~min}\left(\right.$ at $230^{\circ} \mathrm{C}$ and $5 \mathrm{~kg}$ ) was purchased from Solvay (Shanghai), China. N,N-Dimethylformamide (DMF, AR grade) and urea (AR grade) were both produced by Xilong Chemical Co. Ltd, Shantou, China.

\subsection{Preparation of $\mathrm{f}-\mathrm{SiC}$ nanowires}

The $\mathrm{f}-\mathrm{SiC}$ nanowires were obtained by means of ball milling. $1 \mathrm{~g}$ SiC nanowires, $60 \mathrm{~g}$ urea, and $36 \mathrm{ml}$ distilled water were premixed together, and were then ball-milled at $500 \mathrm{rpm}$ for $12 \mathrm{~h}$ with a ratio of grinding media to material approximating $1: 1$. After ball milling, the collected f-SiC nanowires were centrifuged with distilled water for five times to remove the residual urea. The washed $\mathrm{f}-\mathrm{SiC}$ nanowires were collected for use after freeze drying.

\subsection{Preparation of $\mathrm{PVDF} / \mathrm{SiC}$ and $\mathrm{PVDF} / \mathrm{f}-\mathrm{SiC}$ nanowires composites}

Both PVDF/SiC and PVDF/f-SiC nanowires composites were prepared via solution mixing, which has been well demonstrated to be effective in the literature [29]. The PVDF was dissolved into the DMF at $80^{\circ} \mathrm{C}$ under stirring for $2 \mathrm{~h}$; while both $\mathrm{SiC}$ and $\mathrm{f}-\mathrm{SiC}$ nanowires were dispersed in DMF, respectively, with the aid of stirring and sonication. The suspensions of $\mathrm{SiC}$ or $\mathrm{f}-\mathrm{SiC}$ nanowires were then poured into the PVDF solution, followed by further stirring for $0.5 \mathrm{~h}$. The mixed suspensions were cast on the glass substrate at $80^{\circ} \mathrm{C}$. After dried, the PVDF/SiC and PVDF/ $\mathrm{f}-\mathrm{SiC}$ nanowires composite films with filler loadings of 6,10 , and $13.8 \mathrm{vol} \%$ were obtained, respectively. The composites were named by their volume fraction of $\mathrm{SiC}$ or $\mathrm{f}-\mathrm{SiC}$ nanowires in the form of 'PVDF/ $\mathrm{SiCx}$ ' or 'PVDF/f-SiCx'. For example, PVDF/f-SiC6 denotes PVDF based composites with 6 vol\% $\mathrm{f}-\mathrm{SiC}$ nanowires. 
The cast films were, however, usually featured as high porosity, and the pores can be eliminated via hot pressing [30]. Thus, the cast films were cut into pieces and hot pressed into films again at $230^{\circ} \mathrm{C}$ and $10 \mathrm{MPa}$ using a hot press machine (KY-320IA, Kaiyan machinery Co., Ltd, Dongguan, China). To control the thickness of the film, a circular mold with an inner diameter of $110 \mu \mathrm{m}$ and a thickness of $100 \mu \mathrm{m}$ was used. The mold was sandwiched by two polyimide films and then two smooth steel plates to facilitate the molding and demolding processes. During the hot pressing, the pressure was released followed by returning to $10 \mathrm{MPa}$ again for 3 times to eliminate the possible pores. The thickness of the obtained films was measured by a micrometer caliper. Five different areas were measured and the average thickness of the films was in the range of 91 107 $\mu \mathrm{m}$.

\subsection{Characterization}

Transmission electron microscopy (TEM) observations of both $\mathrm{SiC}$ and $\mathrm{f}-\mathrm{SiC}$ nanowires were conducted on a TEM instrument (JEM-2100F, JEOL, Japan) equipped with an Oxford energy-dispersive X-ray (EDX) spectrometer. The accelerating voltage was $200 \mathrm{kV}$.

X-ray photoelectron spectroscopy (XPS) measurements were performed by using a XPS system (ESCALAB 250Xi, Thermo Fisher Scientific, USA).

Thermogravimetric analysis (TGA) was carried out using a synchronous thermal analyzer (STA 2500 Regulus, Netzsch, Germany). The samples were heated from 30 to $800^{\circ} \mathrm{C}$ at a heating rate of $20^{\circ} \mathrm{C} / \mathrm{min}$ under nitrogen atmosphere.

The cryo-fractured surfaces of both PVDF/SiC and $\mathrm{PVDF} / \mathrm{f}-\mathrm{SiC}$ nanowires composites were gold-sputtered and examined via scanning electron microscopy (SEM, Nova NaoSEM 450, FEI, USA). The accelerating voltage was $10 \mathrm{kV}$.

Dynamic frequency sweep measurements of both $\mathrm{PVDF} / \mathrm{SiC}$ and PVDF/f-SiC nanowires composites were performed on a rotation rheometer (DHR-2, TA, USA) with $25 \mathrm{~mm}$ parallel-plate geometry in an oscillatory mode. Complex viscosity $\left(\eta^{*}\right)$ and storage modulus $\left(G^{\prime}\right)$ were measured at $230^{\circ} \mathrm{C}$ under a flow of nitrogen as functions of angular frequency $(\omega)$ ranging from 0.01 to $10 \mathrm{rad} / \mathrm{s}$. A strain sweep was performed prior to each measurement, and the chosen strain of $1 \%$ was well in the linear viscoelastic regime. The gap was chosen to be $0.9 \mathrm{~mm}$ for all measurements.
Fourier transform infrared spectroscopy (FT-IR) spectra were recorded using a FT-IR spectrometer (Vertex 70 , Bruker, Germany) equipped with an attenuated total reflectance (ATR) cell. The scanning range was from 4000 to $600 \mathrm{~cm}^{-1}$ with resolution of $4 \mathrm{~cm}^{-1}$.

The dielectric properties of both $\mathrm{PVDF} / \mathrm{SiC}$ and PVDF/f-SiC nanowires composites were obtained via a TZDM-200-300 LCR meter at frequencies ranging from $100 \mathrm{~Hz}$ to $1 \mathrm{MHz}$ at room temperature. Four specimens were tested for each sample. For depositing the electrodes, the specimens $\left(15 \times 15 \mathrm{~mm}^{2}\right)$ were sandwiched by two multi-orifice plates and were gold-sputtered using a vacuum coating apparatus (Q150R S, Quorum, UK). The diameter of the electrodes was $8 \mathrm{~mm}$.

The out-of-plane thermal conductivity of both PVDF/ $\mathrm{SiC}$ and PVDF/f-SiC nanowires composites was determined by using a NanoFlash instrument (LFA 467, Netzsch, Germany), calculated by Equation (1):

$\lambda=\alpha \rho C_{\mathrm{p}}$

where $\alpha, \rho$, and $C_{\mathrm{p}}$ are the thermal diffusivity, density, and the specific heat capacity of the specimens, respectively. Specimens with diameters of $12.7 \mathrm{~mm}$ and thickness of $1 \mathrm{~mm}$ were prepared via hot pressing. The $\rho$ is based on the formula of $\rho=m / V$, in which $m$ and $V$ are the mass and volume of the samples, respectively. $C_{\mathrm{p}}$ is measured by using a differential scanning calorimetry (DSC) instrument (Q2000, TA, USA) with the sapphire method. Three specimens were tested for each sample.

\section{Results and discussion}

\subsection{Structural analysis of both $\mathrm{SiC}$ and $\mathrm{f}-\mathrm{SiC}$ nanowires}

Figure 1a shows the low resolution TEM micrograph of a $\mathrm{SiC}$ nanowire, and the high resolution TEM micrograph is inserted in its top-right corner. From the high resolution TEM micrograph, one can observe ordered lattice fringes on the surface of $\mathrm{SiC}$ nanowire, which correspond to the $\mathrm{SiC}$ crystals oriented along the $<100>$ axis [31]. Figures $1 \mathrm{~b}$ and $1 \mathrm{c}$ show the corresponding EDX mapping images from Figure 1a. Both $\mathrm{Si}$ and $\mathrm{C}$ elements are clearly observed to be densely distributed along and across the $\mathrm{SiC}$ nanowire. For the f-SiC nanowire, one observes coverings on its surface from the low resolution TEM micrograph (Figure 2a). Due to the presence of coverings on its surface, the ordered lattice fringes can 


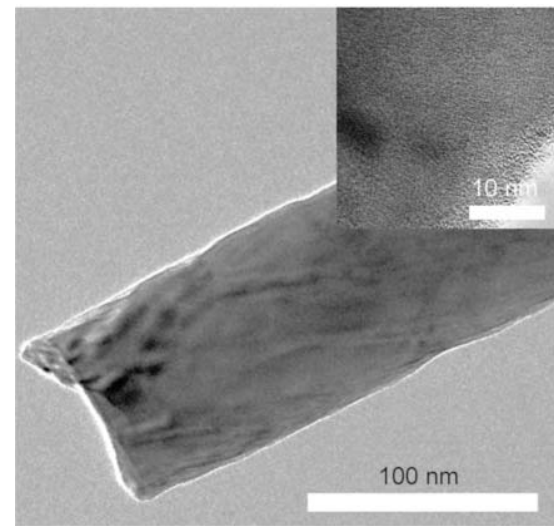

a)

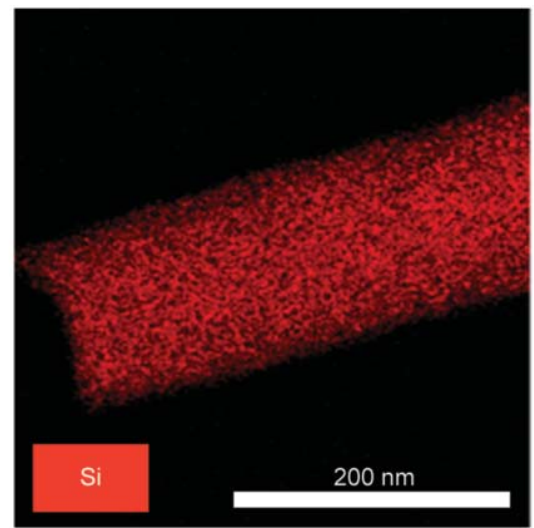

b)

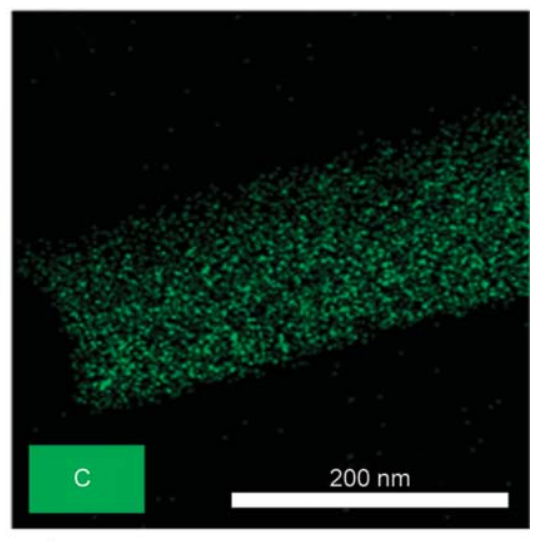

c)

Figure 1. (a) TEM micrographs of $\mathrm{SiC}$ nanowire; (b) and (c) corresponding EDX elemental mapping images of $\mathrm{Si}$ and $\mathrm{C}$, respectively.

hardly be observed in the high resolution TEM micrograph of $\mathrm{f}-\mathrm{SiC}$ nanowire. From the preparation process of $\mathrm{f}-\mathrm{SiC}$ nanowires, it can be deduced that the coverings are urea that are decorated on their surfaces via physical absorption during the ball milling. Correspondingly, the EDX mapping images of f-SiC nanowire reflect the existence of the $N$ element (Figure $2 \mathrm{~d}$ ), in addition to the $\mathrm{Si}$ and $\mathrm{C}$ elements (Figures $2 \mathrm{~b}$ and $2 \mathrm{c}$ ). The $N$ element can be attributed to the $-\mathrm{NH}_{2}$ groups of the coverings (urea).

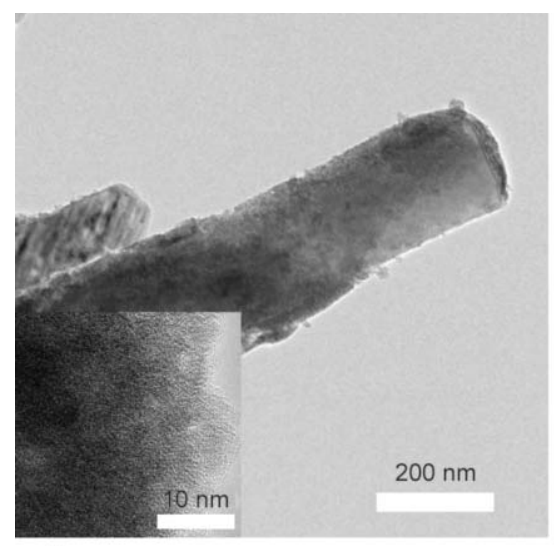

a)

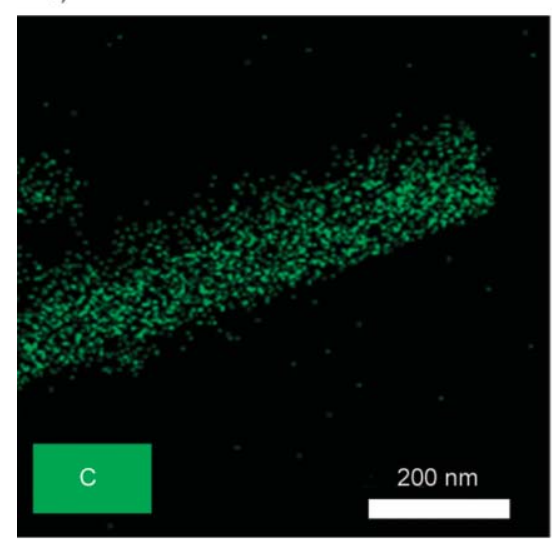

c)
The XPS survey spectra of both $\mathrm{SiC}$ and f-SiC nanowires are shown in Figure 3a. The $\mathrm{SiC}$ nanowires exhibit three main peaks at 102.2, 154.0, and $284.5 \mathrm{eV}$, corresponding to the Si2p, Si2s, and C1s [32], respectively. While the $\mathrm{f}-\mathrm{SiC}$ exhibits two additional peaks at 400.4 and $528.3 \mathrm{eV}$, which correspond to the N1s and $\mathrm{O} 1 \mathrm{~s}$, respectively. This further confirms the successful decoration of the $\mathrm{SiC}$ nanowires surfaces with urea. Figure $3 \mathrm{~b}$ shows the TGA curves of both $\mathrm{SiC}$ and $\mathrm{f}-\mathrm{SiC}$ nanowires. Due to highly thermostable

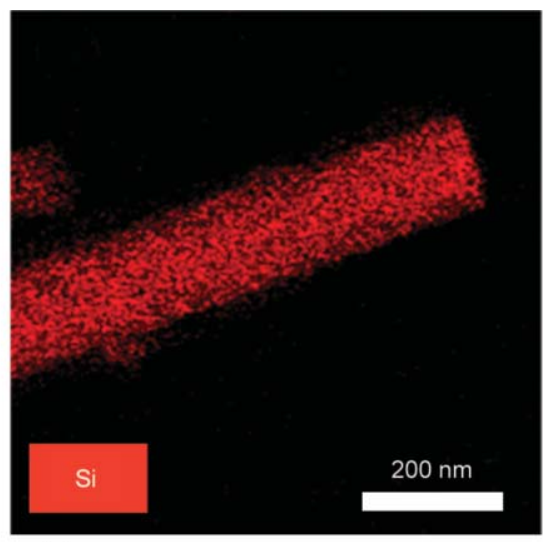

b)

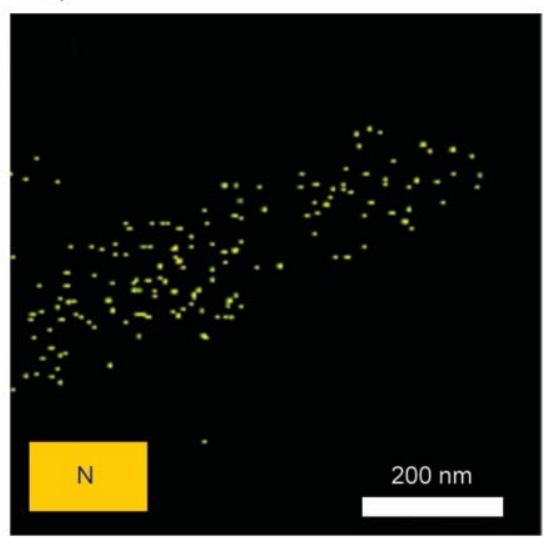

d)

Figure 2. (a) TEM micrographs of f-SiC nanowire; (b-d) corresponding EDX elemental mapping images of Si, C, and N, respectively. 

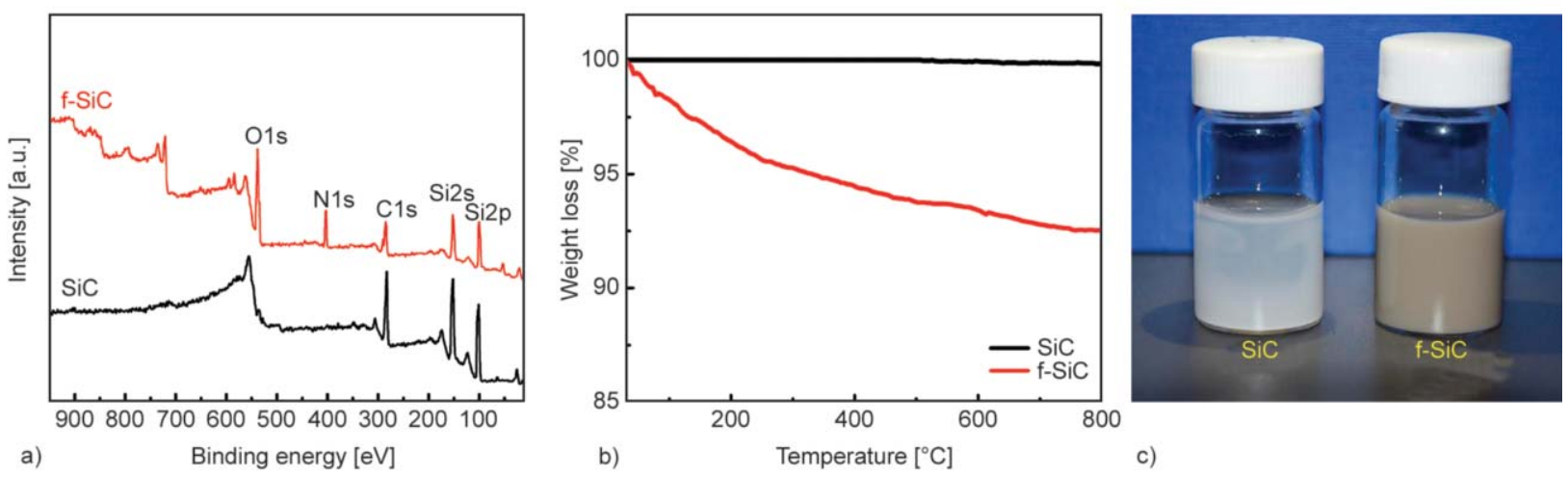

Figure 3. (a) XPS spectra, (b) TGA curves, and (c) photos of both SiC and f-SiC nanowires.

nature, the $\mathrm{SiC}$ nanowires show only a $0.2 \%$ mass loss at $800{ }^{\circ} \mathrm{C}$. However, the $\mathrm{f}-\mathrm{SiC}$ nanowires exhibit gradual mass loss, and a total mass loss of $7.5 \%$ is observed at $800^{\circ} \mathrm{C}$. This can mainly be ascribed to the sequential decomposition of the urea on their surfaces. Decorating the $\mathrm{SiC}$ nanowires with urea can modify their surface property. Due to the existence of $-\mathrm{NH}_{2}$ groups, $\mathrm{f}-\mathrm{SiC}$ nanowires can be dispersed well in the DMF (Figure 3c), and the dispersed suspension can be stable up to two weeks. This can be ascribed to the polar interaction between $\mathrm{f}-\mathrm{SiC}$ nanowires and DMF [33]. However, the SiC nanowires are hard to be dispersed in the DMF, and they precipitate in a short time (Figure 3c), due to the lack of $-\mathrm{NH}_{2}$ groups on their surfaces.

\subsection{Microstructures of both $\mathrm{PVDF} / \mathrm{SiC}$ and $\mathrm{PVDF} / \mathrm{f}-\mathrm{SiC}$ nanowires composites}

Figure 4 shows the SEM micrographs of cryo-fractured surfaces of both PVDF/SiC and PVDF/f-SiC nanowires composites. As can be seen, the f-SiC nanowires exhibit better dispersion in the PVDF matrix (Figures 4d-4f) than the $\mathrm{SiC}$ nanowires do (Figures $4 \mathrm{a}-4 \mathrm{c})$, regardless of their loadings. At a loading of $10 \mathrm{vol} \%$, some of the $\mathrm{SiC}$ nanowires tend to interlace with each other (Figure 4b), and their obvious

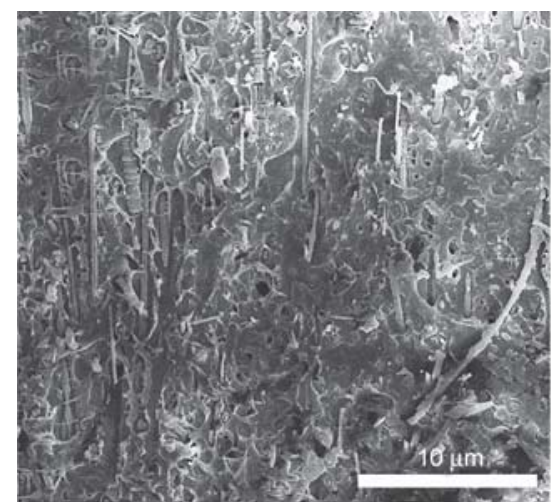

a)

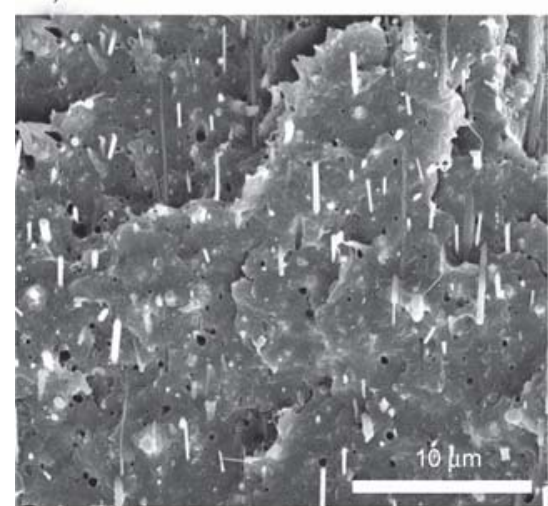

d)

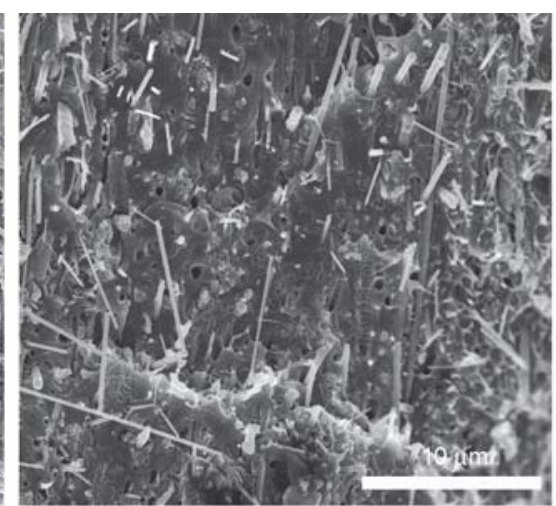

b)

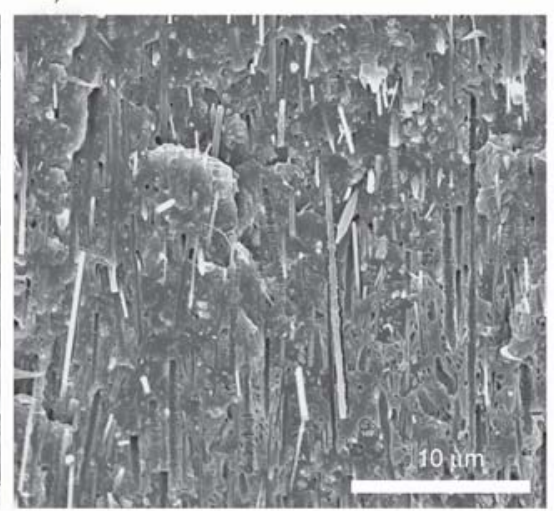

e)

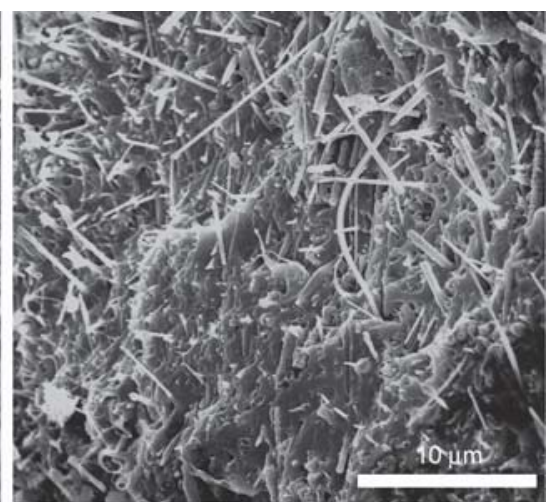

c)

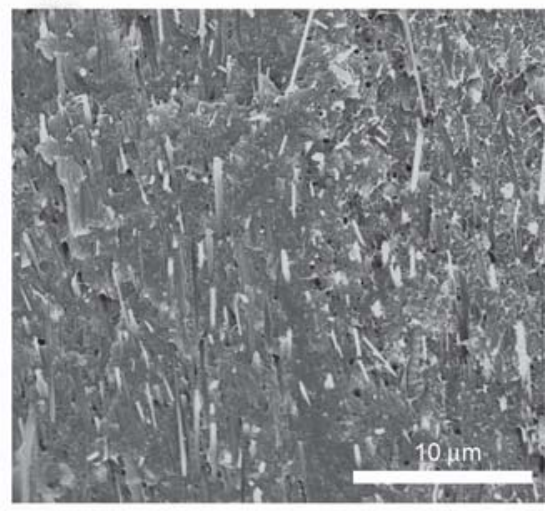

f)

Figure 4. SEM micrographs of $(\mathrm{a}-\mathrm{c}) \mathrm{PVDF} / \mathrm{SiC}$ and $(\mathrm{d}-\mathrm{f}) \mathrm{PVDF} / \mathrm{f}-\mathrm{SiC}$ nanowires composites with filler volume fractions of (a, d) 6, (b, e) 10, and (c, f) $13.8 \mathrm{vol} \%$, respectively. 

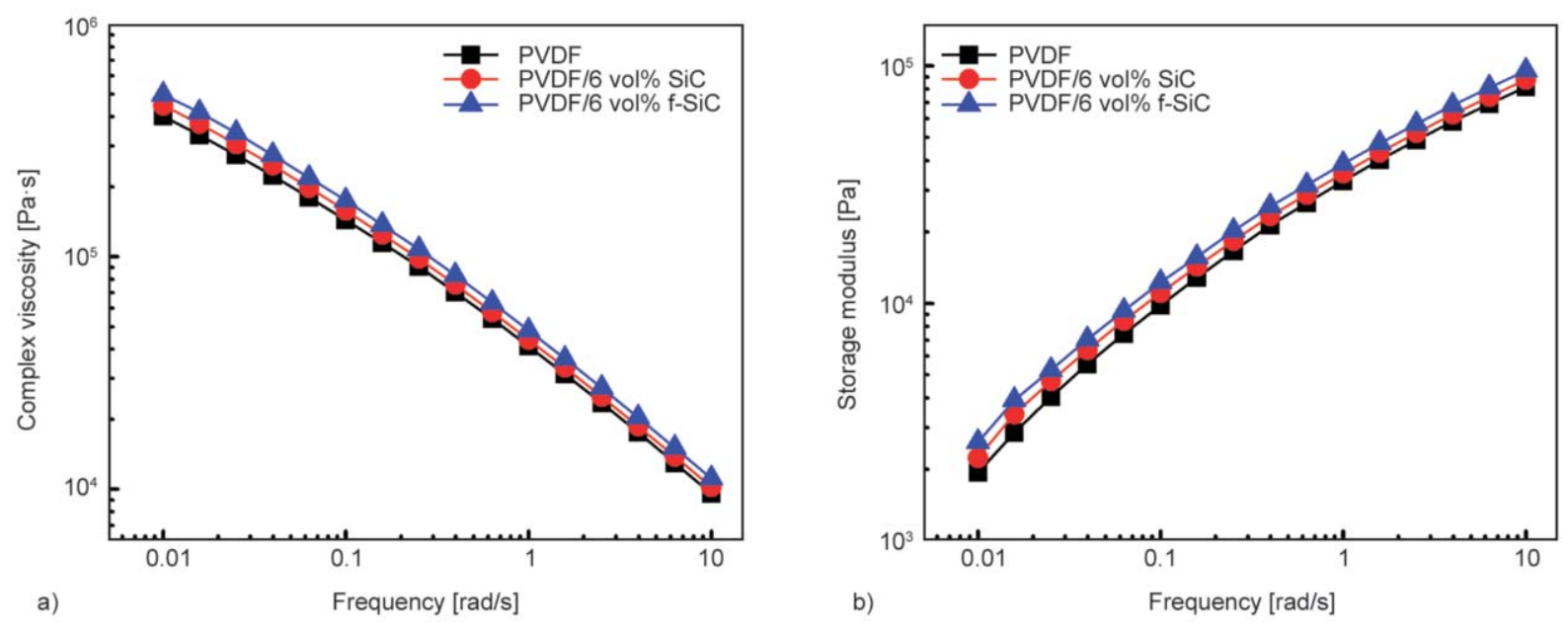

Figure 5. (a) Complex viscosity and (b) storage modulus of neat PVDF, PVDF/SiC6, and PVDF/f-SiC6.

interlacing is observed at a loading of $13.8 \mathrm{vol} \%$ (Figure 4c). However, the $\mathrm{f}-\mathrm{SiC}$ nanowires are tend to disperse parallel in the PVDF matrix, even if at a loading as high as $13.8 \mathrm{vol} \%$ (Figure $4 \mathrm{f}$ ). It is reported that fillers are easier to orient in the flow direction of polymer melt if the interfacial interaction are relatively strong [34]. Herein, the interfacial interaction between PVDF matrix and $\mathrm{f}-\mathrm{SiC}$ nanowires are expected to be enhanced by the surface functionalization. Thus, the $\mathrm{f}-\mathrm{SiC}$ nanowires are easier to orient in the flow direction of PVDF melt during hot pressing. The interfacial interaction between nanofillers and polymer matrix can be evaluated by linear rheological behavior of the nanocomposites [35, 36]. At a given loading of nanofillers, the nanocomposites with stronger interfacial interaction usually exhibit higher $\eta^{*}$ and $G^{\prime}$ than their counterparts with weak interfacial interaction. As shown in Figure 5, PVDF/ f-SiC6 shows higher $\eta^{*}$ and $G^{\prime}$ than those of the $\mathrm{PVDF} / \mathrm{SiC6}$ in the frequency range of 0.01 to $10 \mathrm{rad} / \mathrm{s}$. Thus, it is believed that the $\mathrm{f}-\mathrm{SiC}$ nanowires exhibit stronger interaction with the PVDF matrix than the SiC nanowires do. Both $\eta^{*}$ and $G^{\prime}$ curves of PVDF-based composites with higher loadings of $\mathrm{SiC} / \mathrm{f}-\mathrm{SiC}$ nanowires are not shown, since their $\eta^{*}$ are too high and out of the upper range limit $\left(10^{6} \mathrm{~Pa} \cdot \mathrm{s}\right)$ at low frequency of the rheometer.

The enhanced interfacial interaction between $\mathrm{f}-\mathrm{SiC}$ and PVDF can mainly be deduced to be the formation of hydrogen bonding between $-\mathrm{NH}_{2}$ groups and $\mathrm{F}$ atoms. This can be confirmed by evaluating the changes of binding energies of the $\mathrm{N}$ and $\mathrm{F}$ atoms through XPS spectroscopy. Figures $6 \mathrm{a}$ and $6 \mathrm{~b}$ show the N1s spectra of f-SiC and PVDF/f-SiC13.8, respectively. The curve fitting shows two peaks at 400.6 and $399.7 \mathrm{eV}$ for the N1s spectrum of $\mathrm{f}-\mathrm{SiC}$; while they shift to 400.1 and $399.2 \mathrm{eV}$, respectively, for the N1s spectrum of PVDF/f-SiC13.8. This indicates that electron density of the $\mathrm{N}$ atoms in $\mathrm{PVDF} /$ $\mathrm{f}-\mathrm{SiC} 13.8$ is increased due to the fact that electronenriched $\mathrm{F}$ atoms of the PVDF matrix share their electron cloud with $\mathrm{N}$ nuclei $[37,38]$. Accordingly, the electron density of the $\mathrm{F}$ atoms in the PVDF matrix is decreased as evidenced by the up-shift of F1s
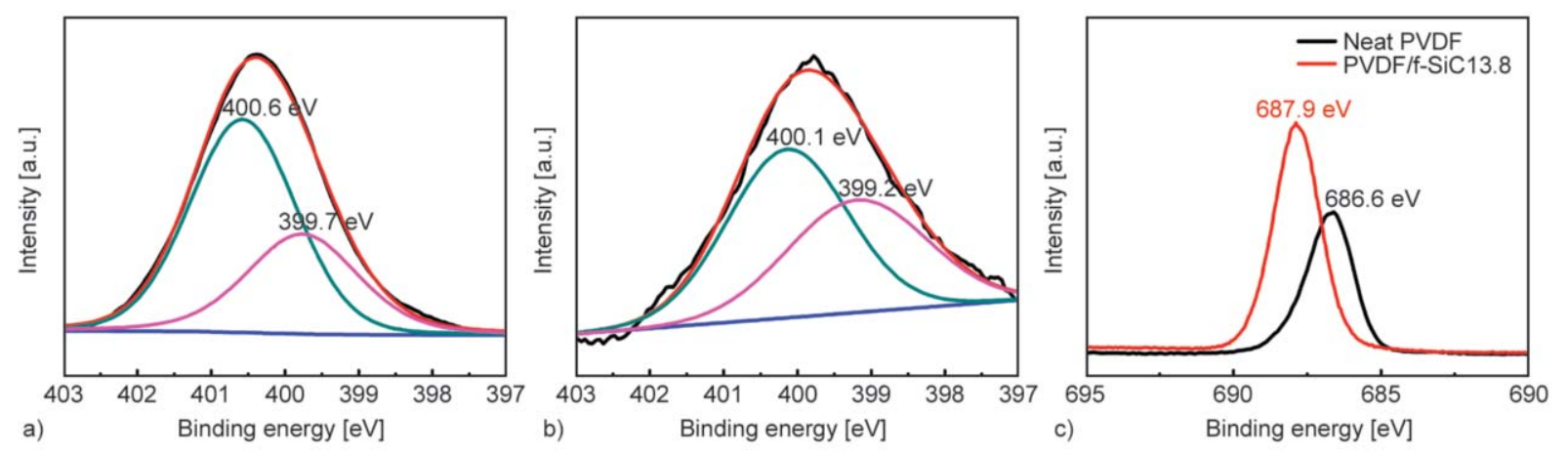

Figure 6. XPS N1s spectra of (a) f-SiC and (b) PVDF/f-SiC13.8; (c) XPS F1s spectra of both neat PVDF and PVDF/f$\mathrm{SiC} 13.8$. 


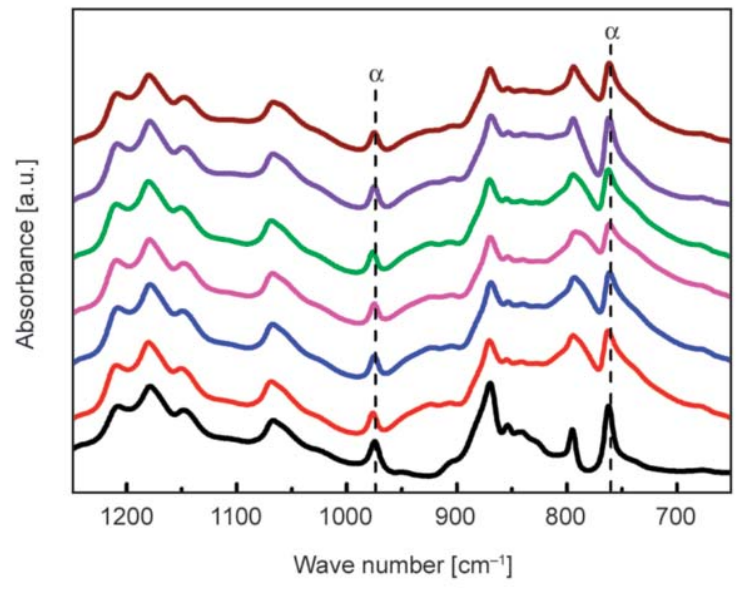

Figure 7. FT-IR spectra of both PVDF/SiC and PVDF/f-SiC nanowires composites with different filler volume fractions.

peak in PVDF/f-SiC13.8 [39], comparing to that of neat PVDF (Figure 6c).

Figure 7 exhibits the ATR FT-IR spectra of both $\mathrm{PVDF} / \mathrm{SiC}$ and $\mathrm{PVDF} / \mathrm{f}-\mathrm{SiC}$ nanowires composites. It is observed that both kind of composites and the neat PVDF mainly show the peaks at 762 and $976 \mathrm{~cm}^{-1}$, which indicates that the non-electroactive $\alpha$ phase is dominant [40]. The absence of electroactive $\beta$ phase in the composites may be due to lack of electrostatic interaction between $-\mathrm{CH}_{2}$ groups of $\mathrm{PVDF}$ and $\mathrm{SiC} / \mathrm{f}-\mathrm{SiC}$ nanowires [41].

\subsection{Dielectric properties}

It is reported that the presence of semi-conductive $\mathrm{SiC}$ nanowires can remarkably enhance the dielectric permittivity of PVDF. However, this usually accompanied with large increase of dielectric loss due to the leakage current caused by the interlacing of $\mathrm{SiC}$ nanowires [42]. In this work, the addition of $\mathrm{SiC}$ nanowires is also observed to enhance the dielectric permittivity of PVDF. The dielectric permittivity increases with the increasing of SiC nanowires' loading in the frequency range of $100 \mathrm{~Hz}$ to $1 \mathrm{MHz}$ (Figure $8 \mathrm{a}$ ). At a SiC nanowires' loading of $13.8 \mathrm{vol} \%$, the dielectric permittivity at $1 \mathrm{kHz}$ of the composite is significantly increased to 42.2 , which is nearly 4 times to that of neat PVDF (10.7). However, the corresponding loss tangent is also significantly increased to 0.2 , which is nearly 10 times to that of neat PVDF (0.022). The remarkable increased loss tangent can be mainly ascribed to the increased leakage current caused by the interlacing of $\mathrm{SiC}$ nanowires (see Figure $4 \mathrm{c}$ ), as evidenced by the higher AC conductivity of PVDF/SiC13.8 (Figure 8c).

High dielectric loss is undesirable for the application of polymer dielectrics in advanced electronics and energy storage. Thus, it is essential to lower the dielectric loss of PVDF/SiC nanowires composites while maintaining a relatively high dielectric permittivity. This is achieved for the PVDF/f-SiC nanowires composites, as shown in Figures $8 \mathrm{a}$ and $8 \mathrm{~b}$. Different from the PVDF/SiC13.8, the PVDF/f-SiC13.8 exhibits a much lower loss $(0.048)$ at $1 \mathrm{kHz}$, which is caused by a nearly one order of magnitude lower AC conductivity at $1 \mathrm{kHz}$ (Figure $8 \mathrm{c}$ ), since the $\mathrm{f}-\mathrm{SiC}$ nanowires are parallelly dispersed in the $\mathrm{PVDF} /$ f-SiC13.8 (Figure 4f). Moreover, the PVDF/f-SiC13.8 still exhibits a nearly 2-fold increased dielectric permittivity (23.3) at $1 \mathrm{kHz}$ compared to that of neat PVDF. The increased dielectric permittivity of PVDF/ f-SiC13.8 can be ascribed to the Maxwell-WagnerSillars effect as well as the formation of micro capacitors between two adjacent paralleled $\mathrm{f}-\mathrm{SiC}$ nanowires $[28,42]$.

\subsection{Thermal conductivity}

Figure 9 shows the thermal conductivity of both $\mathrm{PVDF} / \mathrm{SiC}$ and PVDF/f-SiC nanowires composites. It is observed that the addition of both $\mathrm{SiC}$ and $\mathrm{f}-\mathrm{SiC}$ nanowires enhances the thermal conductivity of the
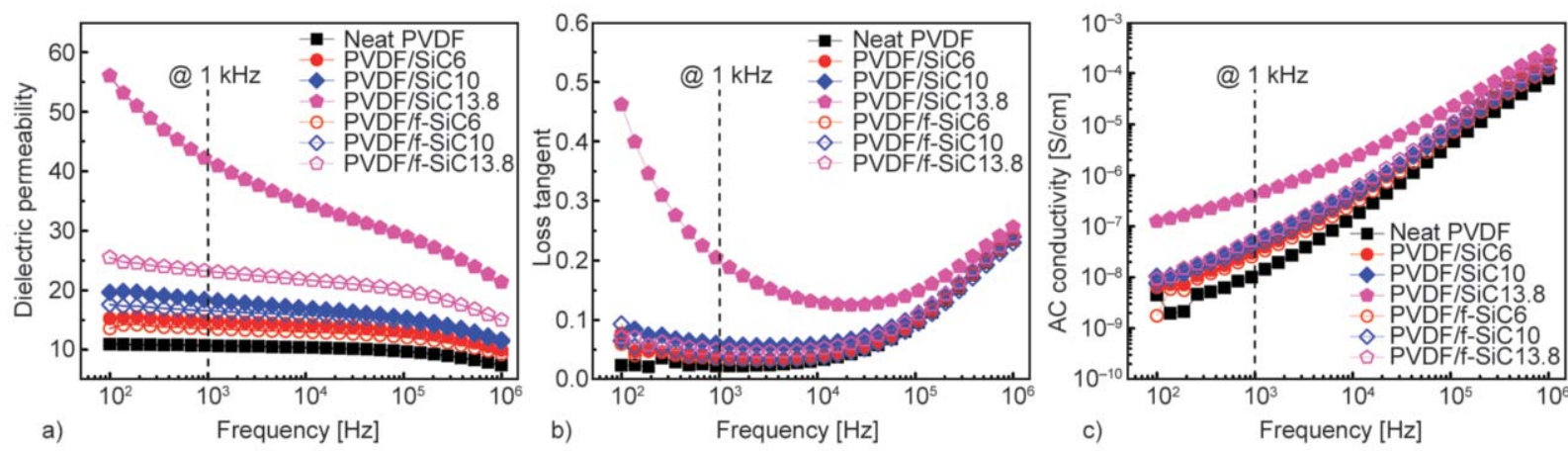

Figure 8. (a) Dielectric permittivity, (b) loss tangent, and (c) C conductivity of neat PVDF, PVDF/SiC, and PVDF/f-SiC nanowires composites. 
PVDF. The increased thermal conductivity can be mainly attributed to the high thermal conductivity nature of the $\mathrm{SiC}$ nanowires [43, 44]. Composites with higher loading of $\mathrm{SiC}$ or $\mathrm{f}-\mathrm{SiC}$ nanowires exhibit higher thermal conductivity. The interlacing of $\mathrm{SiC}$ nanowires can help to enhance the transporting of phonons. Thus, PVDF/SiC10 and PVDF/SiC13.8 show higher thermal conductivity than their PVDF/f$\mathrm{SiC}$ counterparts.

The theoretical thermal conductivity of PVDF/SiC nanowires composites is calculated according to the effective medium model $[25,27]$, and the results is also shown in Figure 9. It is observed that the theoretical thermal conductivity is lower than the experimental one at each loading of both $\mathrm{SiC}$ and $\mathrm{f}-\mathrm{SiC}$ nanowires. The reason is that the nanofillers are assumed to be spherical in the effective medium model, which leads to lower shape factor than that of the nanofillers with high aspect ratios [27].

The thermally conductive, high dielectric permittivity, and low loss PVDF/f-SiC nanowires composites may find potential applications in capacitors for microelectronics rather than energy storage capacitors. For energy storage capacitors, the loss tangent should be at least one order of magnitude lower than the observed one in this work [45]. Moreover, the addition of $\mathrm{SiC} / \mathrm{f}-\mathrm{SiC}$ nanowires would lead to the decreased breakdown strength. As to the capacitors for microelectronics, however, the composites may find potential applications in the future, if the surfacants for modifying the $\mathrm{SiC}$ nanowires can block leakage current more effectively but influence the thermal conductivity moderately.

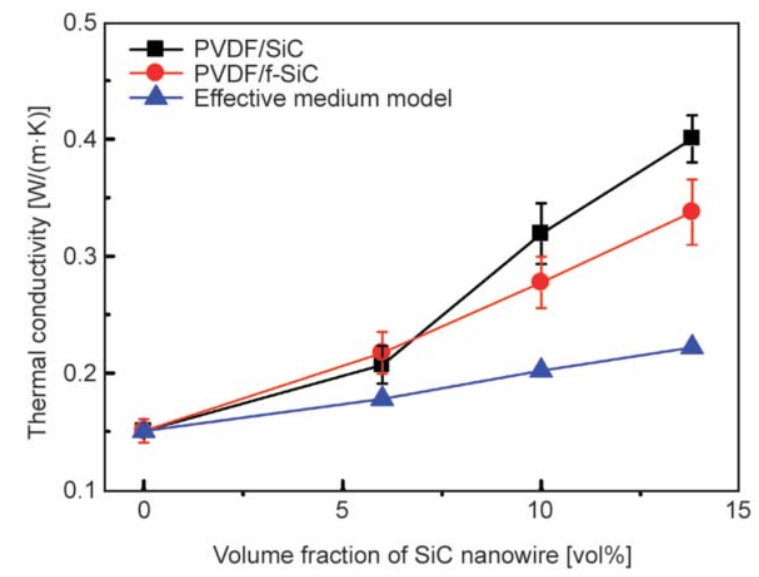

Figure 9. Experimental and theoretical calculated thermal conductivity of PVDF/SiC and PVDF/f-SiC nanowires composites.

\section{Conclusions}

The addition of SiC nanowires into PVDF could enhance the dielectric permittivity, and a significant increase of the dielectric permittivity was achieved with the $\mathrm{SiC}$ nanowires loading of $13.8 \mathrm{vol} \%$. However, the dielectric loss was also remarkably increased, due to the increased leakage current caused by the interlacing of $\mathrm{SiC}$ nanowires. Thus, the $\mathrm{SiC}$ nanowires were amino-functionalized via ball-milling together with the urea for the sake of achieving better dispersion in the PVDF matrix. The enhanced interfacial interaction between the functionalized $\mathrm{SiC}$ (f-SiC) nanowires and PVDF matrix facilitated the orientation of $\mathrm{f}-\mathrm{SiC}$ nanowires during hot pressing. As a result, the interlacing of $\mathrm{f}-\mathrm{SiC}$ nanowires was avoided even at a loading as high as $13.8 \mathrm{vol} \%$. The dielectric loss at $1 \mathrm{kHz}$ of the PVDF/f-SiC13.8 was thus maintained at a low value (0.048), while the dielectric permittivity at $1 \mathrm{kHz}$ was still nearly 2 -fold of that of neat PVDF. Due to relatively high loading of the $\mathrm{f}-\mathrm{SiC}$ nanowires, the PVDF/f-SiC13.8 exhibited a thermal conductivity that was 2-fold of that of the neat PVDF. The thermally conductive, high dielectric permittivity, and low loss $\mathrm{PVDF} / \mathrm{f}-\mathrm{SiC}$ nanowires composites may find potential applications in capacitors for microelectronics.

\section{Acknowledgements}

This work is financially supported by the National Natural Science Foundation of China (Grant Nos. 51605086, 21764001), Guangdong Provincial Natural Science Foundation (No. 2015A030310443), and the Major Program of Natural Science Foundation of Jiangxi, China (No. 20133ACB21006).

\section{References}

[1] Prateek, Thakur V. K., Gupta R. K.: Recent progress on ferroelectric polymer-based nanocomposites for high energy density capacitors: Synthesis, dielectric properties, and future aspects. Chemical Reviews, 116, 42604317 (2016).

https://doi.org/10.1021/acs.chemrev.5b00495

[2] Tjong S. C.: Polyvinylidene fluoride: A versatile polymer for biomedical, electronic, energy and environmental applications. Express Polymer Letters, 12, 395 (2018). https://doi.org/10.3144/expresspolymlett.2018.33

[3] Tang H., Zhou Z., Sodano H. A.: Relationship between $\mathrm{BaTiO}_{3}$ nanowire aspect ratio and the dielectric permittivity of nanocomposites. ACS Applied Materials and Interfaces, 6, 5450-5455 (2014). https://doi.org/10.1021/am405038r 
[4] Guo Q., Xue Q., Wu T., Pan X., Zhang J., Li X., Zhu L.: Excellent dielectric properties of PVDF-based composites filled with carbonized PAN/PEG copolymer fibers. Composites Part A: Applied Science and Manufacturing, 87, 46-53 (2016).

https://doi.org/10.1016/j.compositesa.2016.04.008

[5] Wang B., Ma Y., Na B., Lv R., Liu H., Li W., Zhou H.: Enhanced dielectric thermal stability and permittivity of flexible composite films based on $\mathrm{BaTiO}_{3}$ nanoparticles highly filled PVDF/PAN blend nanofibrous membranes. Polymer Composites, 39, E1841-E1848 (2018). https://doi.org/10.1002/pc.24829

[6] Feng Y., Miao B., Gong H., Xie Y., Wei X., Zhang Z.: High dielectric and mechanical properties achieved in cross-linked PVDF/ $\alpha$-SiC nanocomposites with elevated compatibility and induced polarization at the interface. ACS Applied Materials and Interfaces, 8, 19054 19065 (2016).

https://doi.org/10.1021/acsami.6b04776

[7] Zhou T., Zha J-W., Cui R-Y., Fan B-H., Yuan J-K., Dang Z-M.: Improving dielectric properties of $\mathrm{BaTiO}_{3} /$ ferroelectric polymer composites by employing surface hydroxylated $\mathrm{BaTiO}_{3}$ nanoparticles. ACS Applied Materials and Interfaces, 3, 2184-2188 (2011). https://doi.org/10.1021/am200492q

[8] Chanmal C. V., Jog J. P.: Dielectric relaxations in PVDF/BaTiO3 nanocomposites. Express Polymer Letters, 2, 294-301 (2008).

https://doi.org/10.3144/expresspolymlett.2008.35

[9] Hao Y. N., Wang X. H., O’Brien S., Lombardi J., Li L. T.: Flexible $\mathrm{BaTiO}_{3} / \mathrm{PVDF}$ gradated multilayer nanocomposite film with enhanced dielectric strength and high energy density. Journal of Materials Chemistry C, 3, 9740-9747 (2015). https://doi.org/10.1039/C5TC01903F

[10] Rekik H., Ghallabi Z., Royaud I., Arous M., Seytre G., Boiteux G., Kallel A.: Dielectric relaxation behaviour in semi-crystalline polyvinylidene fluoride (PVDF)/ $\mathrm{TiO}_{2}$ nanocomposites. Composites Part B: Engineering, 45, 1199-1206 (2013).

https://doi.org/10.1016/j.compositesb.2012.08.002

[11] Hu P., Jia Z., Shen Z., Wang P., Liu X.: High dielectric constant and energy density induced by the tunable $\mathrm{TiO}_{2}$ interfacial buffer layer in PVDF nanocomposite contained with core-shell structured TiO $\mathrm{B}_{2} \mathrm{BaTiO}_{3}$ nanoparticles. Applied Surface Science, 441, 824-831 (2018). https://doi.org/10.1016/j.apsusc.2018.02.112

[12] Gaur A. M., Rana D. S.: Structural, optical and electrical properties of $\mathrm{MgCl}_{2}$ doped polyvinylidene fluoride (PVDF) composites. Journal of Materials Science: Materials in Electronics, 26, 1246-1251 (2015). https://doi.org/10.1007/s10854-014-2533-7

[13] Gaur A. M., Rana D. S.: Effect of $\mathrm{CoCl}_{2}-\mathrm{BaCl}_{2}$ fillers on morphology, dielectric constant and conductivity of PVDF composite for pressure sensing application. Journal of Materials Science: Materials in Electronics, 27, 2293-2299 (2016).

https://doi.org/10.1007/s10854-015-4024-x
[14] An A. L., Boggs S. A., Calame J.: Energy storage in polymer films with high dielectric constant fillers. IEEE Electrical Insulation Magazine, 24, 5-10 (2008). https://doi.org/10.1109/MEI.2008.4591430

[15] Zhu L.: Exploring strategies for high dielectric constant and low loss polymer dielectrics. Journal of Physical Chemistry Letters, 5, 3677-3687 (2014).

https://doi.org/10.1021/jz501831q

[16] Toor A., So H., Pisano A. P.: Improved dielectric properties of polyvinylidene fluoride nanocomposite embedded with poly(vinylpyrrolidone)-coated gold nanoparticles. ACS Applied Materials and Interfaces, 9, 6369-6375 (2017).

https://doi.org/10.1021/acsami.6b13900

[17] Zhang Y., Wang Y., Deng Y., Li M., Bai J.: Enhanced dielectric properties of ferroelectric polymer composites induced by metal-semiconductor $\mathrm{Zn}-\mathrm{ZnO}$ coreshell structure. ACS Applied Materials and Interfaces, 4, 65-68 (2012).

https://doi.org/10.1021/am2016156

[18] Yuan J-K., Yao S-H., Dang Z-M., Sylvestre A., Genestoux M., Bai J.: Giant dielectric permittivity nanocomposites: Realizing true potential of pristine carbon nanotubes in polyvinylidene fluoride matrix through an enhanced interfacial interaction. Journal of Physical Chemistry C, 115, 5515-5521 (2011).

https://doi.org/10.1021/jp1117163

[19] Sharma M., Srinivas V., Madras G., Bose S.: Outstanding dielectric constant and piezoelectric coefficient in electrospun nanofiber mats of PVDF containing silver decorated multiwall carbon nanotubes: Assessing through piezoresponse force microscopy. RSC Advances, 6, 6251-6258 (2016). https://doi.org/10.1039/C5RA25671B

[20] Rahman M. A., Chung G-S.: Synthesis of PVDFgraphene nanocomposites and their properties. Journal of Alloys and Compounds, 581, 724-730 (2013). https://doi.org/10.1016/j.jallcom.2013.07.118

[21] Chen J-J., Li Y., Zheng X-M., He F-A., Lam K-H.: Enhancement in electroactive crystalline phase and dielectric performance of novel PEG-graphene/PVDF composites. Applied Surface Science, 448, 320-330 (2018). https://doi.org/10.1016/j.apsusc.2018.04.144

[22] Yuan J.: Percolation of carbon nanomaterials for high-k polymer nanocomposites. Chinese Chemical Letters, 28, 2036-2044 (2017). https://doi.org/10.1016/j.cclet.2017.08.020

[23] Wu C., Huang X., Wu X., Yu J., Xie L., Jiang P.: $\mathrm{TiO}_{2}-$ nanorod decorated carbon nanotubes for high-permittivity and low-dielectric-loss polystyrene composites. Composites Science and Technology, 72, 521-527 (2012). https://doi.org/10.1016/j.compscitech.2011.12.014

[24] Zhou T., Zha J-W., Hou Y., Wang D., Zhao J., Dang Z-M.: Surface-functionalized MWNTs with emeraldine base: Preparation and improving dielectric properties of polymer nanocomposites. ACS Applied Materials and Interfaces, 3, 4557-4560 (2011). https://doi.org/10.1021/am201454e 
[25] Yuan J., Yao S., Li W., Sylvestre A., Bai J.: Anisotropic percolation of $\mathrm{SiC}$-carbon nanotube hybrids: A new route toward thermally conductive high-k polymer composites. Journal of Physical Chemistry C, 121, 12063 12070 (2017).

https://doi.org/10.1021/acs.jpcc.7b03372

[26] Kuang J., Cao W.: Stacking faults induced high dielectric permittivity of SiC wires. Applied Physics Letters, 103, 112906/1-112906/4 (2013). https://doi.org/10.1063/1.4821036

[27] Zhou W., Chen Q., Sui X., Dong L., Wang Z.: Enhanced thermal conductivity and dielectric properties of $\mathrm{Al} /$ $\beta$-SiCw/PVDF composites. Composites Part A: Applied Science and Manufacturing, 71, 184-191 (2015). https://doi.org/10.1016/j.compositesa.2015.01.024

[28] Bi J., Gu Y., Zhang Z., Wang S., Li M., Zhang Z.: Coreshell $\mathrm{SiC} / \mathrm{SiO}_{2}$ whisker reinforced polymer composite with high dielectric permittivity and low dielectric loss. Materials and Design, 89, 933-940 (2016). https://doi.org/10.1016/j.matdes.2015.10.050

[29] Ribeiro C., Costa C. M., Correia D. M., Nunes-Pereira J., Oliveira J., Martins P., Gonçalves R., Cardoso V. F., Lanceros-Méndez S.: Electroactive poly(vinylidene fluoride)-based structures for advanced applications. Nature Protocols, 13, 681-704 (2018). https://doi.org/10.1038/nprot.2017.157

[30] Sencadas V., Filho R. G., Lanceros-Mendez S.: Processing and characterization of a novel nonporous poly (vinilidene fluoride) films in the $\beta$ phase. Journal of NonCrystalline Solids, 352, 2226-2229 (2006).

https://doi.org/10.1016/j.jnoncrysol.2006.02.052

[31] Nakano H., Watari K., Kinemuchi Y., Ishizaki K., Urabe K.: Microstructural characterization of high-thermalconductivity $\mathrm{SiC}$ ceramics. Journal of the European Ceramic Society, 24, 3685-3690 (2004).

https://doi.org/10.1016/j.jeurceramsoc.2003.12.019

[32] Lei W-W., Li H., Shi L-Y., Diao Y-F., Zhang Y-L., Ran R., Ni W.: Achieving enhanced hydrophobicity of graphene membranes by covalent modification with polydimethylsiloxane. Applied Surface Science, 404, 230-237 (2017).

https://doi.org/10.1016/j.apsusc.2017.01.292

[33] Cho S., Lee J. S., Jang J.: Poly(vinylidene fluoride)/ $\mathrm{NH}_{2}$-treated graphene nanodot/reduced graphene oxide nanocomposites with enhanced dielectric performance for ultrahigh energy density capacitor. ACS Applied Materials and Interfaces, 7, 9668-9681 (2015).

https://doi.org/10.1021/acsami.5b01430

[34] Gorga R. E., Lau K. K. S., Gleason K. K., Cohen R. E.: The importance of interfacial design at the carbon nanotube/polymer composite interface. Journal of Applied Polymer Science, 102, 1413-1418 (2006).

https://doi.org/10.1002/app.24272

[35] Wang H., Yang X., Fu Z., Zhao X., Li Y., Li J.: Rheology of nanosilica-compatibilized immiscible polymer blends: Formation of a 'heterogeneous network' facilitated by interfacially anchored hybrid nanosilica. Macromolecules, 50, 9494-9506 (2017).

https://doi.org/10.1021/acs.macromol.7b02143
[36] Wang B., Peng D., Lv R., Na B., Liu H., Yu Z.: Generic melt compounding strategy using reactive graphene towards high performance polyethylene/graphene nanocomposites. Composites Science and Technology, 177, 1-9 (2019). https://doi.org/10.1016/j.compscitech.2019.04.013

[37] Tang Z., Wu X., Guo B., Zhang L., Jia D.: Preparation of butadiene-styrene-vinyl pyridine rubber-graphene oxide hybrids through co-coagulation process and in situ interface tailoring. Journal of Materials Chemistry, 22, 7492-7501 (2012). https://doi.org/10.1039/c2jm00084a

[38] Salim N. V., Hameed N., Guo Q.: Competitive hydrogen bonding and self-assembly in poly(2-vinyl pyridine)-block-poly(methyl methacrylate)/poly(hydroxyether of bisphenol A) blends. Journal of Polymer Science Part B: Polymer Physics, 47, 1894-1905 (2009). https://doi.org/10.1002/polb.21792

[39] Tao M., Liu F., Xue L.: Hydrophilic poly(vinylidene fluoride) (PVDF) membrane by in situ polymerisation of 2-hydroxyethyl methacrylate (HEMA) and microphase separation. Journal of Materials Chemistry, 22, 9131-9137 (2012).

https://doi.org/10.1039/C2JM30695F

[40] Martins P., Lopes A. C., Lanceros-Mendez S.: Electroactive phases of poly(vinylidene fluoride): Determination, processing and applications. Progress in Polymer Science, 39, 683-706 (2014).

https://doi.org/10.1016/j.progpolymsci.2013.07.006

[41] Martins P., Caparros C., Gonçalves R., Martins P. M., Benelmekki M., Botelho G., Lanceros-Mendez S.: Role of nanoparticle surface charge on the nucleation of the electroactive $\beta$-poly(vinylidene fluoride) nanocomposites for sensor and actuator applications. Journal of Physical Chemistry C, 116, 15790-15794 (2012). https://doi.org/10.1021/jp3038768

[42] Lopes A. C., Costa C. M., Serra R. S., Neves I. C., Gomez Ribelles J. L., Lanceros-Méndez S.: Dielectric relaxation, AC conductivity and electric modulus in poly(vinylidene fluoride)/NaY zeolite composites. Solid State Ionics, 235, 42-50 (2013).

https://doi.org/10.1016/j.ssi.2013.01.013

[43] Slack G. A.: Thermal conductivity of pure and impure silicon, silicon carbide, and diamond. Journal of Applied Physics, 35, 3460-3466 (1964). https://doi.org/10.1063/1.1713251

[44] Li Y., Huang X., Hu Z., Jiang P., Li S., Tanaka T.: Large dielectric constant and high thermal conductivity in poly(vinylidene fluoride)/barium titanate/silicon carbide three-phase nanocomposites. ACS Applied Materials and Interfaces, 3, 4396-4403 (2011). https://doi.org/10.1021/am2010459

[45] Baer E., Zhu L.: 50th anniversary perspective: Dielectric phenomena in polymers and multilayered dielectric films. Macromolecules, 50, 2239-2256 (2017). https://doi.org/10.1021/acs.macromol.6b02669 